\title{
Simultaneous bioethanol distillery (2) CrossMark wastewater treatment and xylanase production by the phyllosphere yeast Pseudozyma antarctica GB-4(0)
}

Takashi Watanabe ${ }^{1,2}$, Ken Suzuki ${ }^{1}$, Ikuo Sato ${ }^{1}$, Tomotake Morita ${ }^{3}$, Hideaki Koike ${ }^{4}$, Yukiko Shinozaki ${ }^{1}$, Hirokazu Ueda ${ }^{1}$, Motoo Koitabashi ${ }^{1}$ and Hiroko K Kitamoto ${ }^{1 *}$

\begin{abstract}
Bioethanol production using lignocellulosic biomass generates lignocellulosic bioethanol distillery wastewater (LBDW) that contains a large amount of xylose, making it a potential inexpensive source of xylose for biomaterials production. The main goal of this study was the production of useful enzymes from LBDW during treatment of this wastewater. In this study, we found that xylose strongly induced two yeast strains, Pseudozyma antarctica T-34 and GB-4(0), to produce novel xylanases, PaXynT and PaXynG, respectively. The nucleotide sequence of PaXynT [accession No. DF196774 (GAC73192.1)], obtained from the genome database of strain T-34 using its N-terminal amino acid sequence, was 91\% identical to that of PaXynG (accession No. AB901085), and the deduced amino acid sequence is $98 \%$ identical. The specific activities of the purified PaXynT and PaXynG were about $52 \mathrm{U} / \mathrm{mg}$. The optimal pH and temperature for both enzymes' activities were 5.2 and $50^{\circ} \mathrm{C}$, respectively. They hydrolyzed xylan to xylose and neither had $\beta$-xylosidase (EC 3.2.1.37) activity, indicating that they are endo- $\beta$-xylanases (EC 3.2.1.8). With these results, we expect that PaXyns can be employed in saccharizing lignocellulosic biomass materials for the production of useful products just like other endoxylanases. After $72 \mathrm{~h}$ of LBDW fed-batch cultivation using a jar-fermentor, strain GB-4(0) produced $17.3 \mathrm{U} / \mathrm{ml}$ (corresponding to about $0.3 \mathrm{~g} / \mathrm{l}$ ) of PaXynG and removed $63 \%$ of dissolved organic carbon and $87 \%$ of dissolved total phosphorus from LBDW. These results demonstrate the potential of $P$. antarctica for xylanase production during LBDW treatment.
\end{abstract}

Keywords: Xylanase, Pseudozyma antarctica, Jar-fermentor, Xylose inducible, Lignocellulosic bioethanol distillery wastewater, Wastewater treatment

\section{Introduction}

Wastewater treatment system that uses yeasts could remove large amounts of organic compounds $(10,000$ COD mg/l/day), requires little space, and discharges little waste sludge (Yoshizawa 1978). This method is useful for treating food, brewing, and beverage industry wastewater (Yoshizawa 1978; Watanabe et al. 2009). Furthermore, the utilization of high strength wastewater is considered

\footnotetext{
*Correspondence: kitamoto@affrc.go.jp

${ }^{1}$ National Institute for Agro-Environmental Sciences (NIAES),

3-1-3 Kannondai, Tsukuba, Ibaraki 305-8604, Japan

Full list of author information is available at the end of the article
}

as an attractive way to produce useful materials, single cell protein, lactic acid, methane, and enzymes (lipase and protease) (Siso 1996; Angenent et al. 2004). Accordingly, using yeasts were suitable for high yield production of useful materials from high strength wastewater (Watanabe et al. 2013a, 2014a).

Producing bioethanol from lignocellulosic biomass generates lignocellulosic bioethanol distillery wastewater (LBDW) that contains high concentrations of organic compounds $(30,000-60,000$ COD $\mathrm{mg} / \mathrm{l})$. The cost of LBDW treatment directly increases the cost of bioethanol production. Since the ethanol-producing yeast Saccharomyces cerevisiae does not ferment xylose

\section{Springer}


(Matsushika et al. 2008), the resulting LBDW is found to contain a large amount of xylose, making it a potential inexpensive source of fermentable xylose.

A non-pathogenic basidiomycetous yeast, Pseudozyma antarctica has an ability to assimilate various kinds of carbon sources (Boekhout 2011). Recently, we found that $P$. antarctica GB-4(0) efficiently removed organic compounds from shochu (a Japanese traditional distilled liquor) distillery wastewater (Watanabe et al. 2013b). The whole genome sequence of $P$. antarctica T-34 had already been determined and annotated (Morita et al. 2013). Moreover, we discovered that $P$. antarctica produces a cutinase-like enzyme (CLE), designated as PaE $(22-\mathrm{kDa})$, which efficiently degrades various synthetic biodegradable polyesters (Kitamoto et al. 2011; Shinozaki et al. 2013), and that the PaE productivity was increased by xylose (Watanabe et al. 2014b). For scaleup production of $\mathrm{PaE}$, under xylose feeding cultivation of $P$. antarctica using a jar fermentor (Watanabe et al. 2014b), we found a highly secreted 33-kDa unknown protein (unpublished data). If this 33-kDa unknown protein is a useful enzyme, it could be efficiently produced from large amounts of cheap xylose containing in LBDW during treatment.

A basidiomycetous yeast, Cryptococcus sp. strain S-2 (Masaki et al. 2012) and a yeast-like fungus, Aureobasidium pullulans (Dobberstein and Emeis 1989) secrete xylanases from xylose. Endo- $\beta$-xylanases (EC 3.2.1.8), which hydrolyze xylopyranosyl linkages of $\beta-1,4-x y l a n$, the principal type of hemicellulose, are useful for saccharizing lignocellulosic biomass materials for the production of bioethanol and other useful chemicals (Biely 1985). Xylanases are also utilized in paper and pulp industries (Pokhrel and Viraraghavan 2004) and pretreatment of animal feeds (Beg et al. 2001). The utilization of LBDW, a cheap substrate for xylanase production, would be an attractive way to reduce xylanase production costs, while treating LBDW.

In this study, we attempted to purify and identify the 33-kDa unknown proteins from $P$. antarctica T-34 and GB-4(0) using the genomic DNA sequence of T-34. After confirming that these proteins were xylanases, their biochemical properties were characterized. The xylanase of GB-4(0) was produced from LBDW derived from rice straw hydrolysate in large scale using a jar-fermentor.

\section{Materials and methods Strains}

Pseudozyma antarctica GB-4(0) was isolated from rice husks (Kitamoto et al. 2011) whereas P. antarctica T-34 was isolated from Mt. Tsukuba soil sample (Kitamoto et al. 1990). These strains were deposited in the Genebank at the National Institute of Agrobiological Sciences
(NIAS), Japan [accession numbers: T-34, MAFF 306900; GB-4(0), MAFF 306999].

\section{Cultivation conditions}

The two yeast strains were pre-cultivated in test tubes containing $5 \mathrm{ml}$ of YM medium $(0.3 \%$ yeast extract, $0.3 \%$ malt extract, $0.5 \%$ peptone, $1 \%$ dextrose) at $30^{\circ} \mathrm{C}$ with reciprocal shaking at $160 \mathrm{rpm}$ for $24 \mathrm{~h}$. The precultures $(100 \mu \mathrm{l})$ were added to $100 \mathrm{ml}$ flasks containing $20 \mathrm{ml}$ of modified fungal minimum medium (FMM) ( $0.3 \%$ yeast extract, $0.2 \% \mathrm{NaNO}_{3}, 0.06 \% \mathrm{KH}_{2} \mathrm{PO}_{4}, 0.06 \%$ $\mathrm{MgSO}_{4} \cdot 7 \mathrm{H}_{2} \mathrm{O}$ ) with $8 \%$ xylose or beechwood xylan. Xylose and other nutrients were separately autoclaved $\left(121^{\circ} \mathrm{C}, 20 \mathrm{~min}\right)$. The cultures were cultivated at $30^{\circ} \mathrm{C}$ with rotary shaking at $200 \mathrm{rpm}$ for $96 \mathrm{~h}$.

Every $24 \mathrm{~h}, 1-\mathrm{ml}$ aliquots of the culture were harvested and centrifuged at 15,000 rpm for $5 \mathrm{~min}$. The pellets were dried at $105^{\circ} \mathrm{C}$ for $2 \mathrm{~h}$ and their dry cell weights were measured to investigate cell growth. At the same time, the xylanase activities of the supernatant were measured as described below.

\section{Enzyme activity}

Xylanase activity was determined using xylan as the substrate at $30^{\circ} \mathrm{C}$ for $30 \mathrm{~min}$. The reaction mixture contained $15 \mathrm{mM}$ sodium acetate buffer ( $\mathrm{pH} 5.2), 1.0 \%$ (w/v) beechwood xylan (Sigma-Aldrich Japan), and $100 \mu$ l of culture supernatant in a total volume of $1 \mathrm{ml}$. After the reaction, the amount of reducing sugar was analyzed by the modified Somogyi-Nelson method (Hatanaka and Kobara $1980)$ with D-xylose as the standard. One unit (U) of xylanase activity was defined as $1 \mu \mathrm{mol}$ of $\mathrm{D}$-xylose liberated per min in the reaction mixture.

$\beta$-Xylosidase activity was also determined using the synthetic substrate $p$-nitrophenyl- $\beta$-D-xylopyranoside (pNP-X) (Iefuji et al. 1996). The reaction mixture contained $200 \mu \mathrm{l}$ of $4 \mathrm{mM}$ pNP-X solution, $600 \mu \mathrm{l}$ of $30 \mathrm{mM}$ sodium acetate buffer ( $\mathrm{pH} 5.2)$ and $200 \mu \mathrm{l}$ of enzyme solution in a total volume of $1 \mathrm{ml}$. After $30 \mathrm{~min}, 2 \mathrm{ml}$ of $0.2 \mathrm{M}$ Tris- $\mathrm{HCl}$ buffer $(\mathrm{pH} 8.5)$ was added to stop the enzyme reaction. One unit of $\beta$-xylosidase activity was defined as $1 \mu \mathrm{mol}$ of $p$-nitrophenol released per min.

\section{Electrophoresis}

Sodium dodecyl sulfate-polyacrylamide gel electrophoresis (SDS-PAGE) was performed according to the method of Laemmli (1970) using a $14.1 \%$ polyacrylamide slab gel. Proteins were visualized by Coomassie brilliant blue (CBB) staining using Phast Gel Blue R (GE Healthcare) and by silver staining (2D-Silver stain II "Daiichi"; Daiichi Pure Chemicals Co., Ltd. Tokyo, Japan).

The proteins were transferred to PVDF membranes (Pall, Port Washington, NY, USA) by semidry blotting. 
The 33-kDa band corresponding to P. antarctica T-34 protein, which was designated as PaXynT was excised and its N-terminal amino acid sequence was determined using ABI Procise 491HT (Applied Biosystems, Foster City, CA, USA).

\section{Genomic DNA isolation}

The two yeast strains were cultivated in $5 \mathrm{ml}$ of YM medium overnight. The cultures were centrifuged, and genomic DNA was isolated from the cell pellets using Dr. GenTLE for Yeast (Takara, Kyoto, Japan).

\section{Sequencing of the PaXyn genes}

The N-terminal peptide sequence of the PaXynT was searched in the annotated whole genome sequences of P. antarctica T-34 (Morita et al. 2013), and was used to obtain its genomic sequence. To determine the nucleotide sequence of the unknown protein produced by $P$. antarctica GB-4(0), designated as PaXynG, forward primers (PaXynF1, PaXynF2, PaXynF3, and PaXynF4) and reverse primers (PaXynR1, PaXynR2, PaXynR3, and PaXynR4) were designed based on the genomic sequence of PaXynT. The primer sequences and their positions in the T-34 gene are shown in Table 1. The entire gene for PaXynG was obtained by PCR amplification using the above primers and genomic DNA of $P$. antarctica GB-4(0) as template. The reaction was performed using TaqEX (Takara, Kyoto, Japan) according to the manufacturer's instructions. Amplified DNA fragments were subjected to $1 \%$ agarose gel electrophoresis and were purified using QIAquick Gel Extraction Kit (Qiagen, Hilden, Germany). The sequences of the PCR products were determined using BigDye Terminator Cycle Sequence V3.1 Kit with the same primers and Applied Biosystems 3100 Genetic Analyzer (Applied Biosystems, Foster City, CA, USA).

Table 1 Primers used for sequencing of PaXyng gene of P. antarctica GB-4(0)

\begin{tabular}{llr}
\hline $\begin{array}{l}\text { Primer } \\
\text { name }\end{array}$ & Primer sequence & Position $^{\text {a }}$ \\
\hline PaxynF1 & 5'-GAAGGCTGAAGCTTTGGCTCTGACAT-3' & -600 to -574 \\
PaxynF2 & 5'-CATGCTTGAAGCTCCAAGAAGATATAA-3' & -120 to -93 \\
PaxynF3 & 5'-CACTCGCAGCTGCCTTCGTGGGTGCAG-3' & 361 to 387 \\
PaxynF4 & 5'-GAAGGCAGTCTGCTCGGCCGCTCCCGA-3' & 841 to 867 \\
PaxynR1 & 5'-TGTGGTGTTTGTTTGGCGTTTTTGCTT-3' & 0 to -26 \\
PaxynR2 & 5'-ATCCCACGCGTACACCTTGCCCTTGTA-3' & 480 to 453 \\
PaxynR3 & 5'-CCCACGCAGTTGGACTGGGCCAGGCAG-3' & 960 to 933 \\
PaxynR4 & 5'-CGAGCGCGATTTTCTCCGAGTCTAAA-3' & 1,390 to 1,365 \\
\hline
\end{tabular}

a Positions of the nucleotides in the PaXynT gene sequence of $P$. antarctica T-34.

\section{Purification}

After $96 \mathrm{~h}$ flask cultivation of both strains, the culture supernatants obtained after centrifugation $(7,000 \times g$, $10 \mathrm{~min}, 4^{\circ} \mathrm{C}$ ) were filtered using a membrane filter (ADVANTEC ${ }^{\circ}$ C045A090C, $0.45 \mu \mathrm{m}$, Toyo Roshi Kaisha, Ltd. Japan). One-ml aliquots of filtrates were applied to a TSK-GEL3000SW $\mathrm{XL}_{\mathrm{XL}}$ column (Tosoh) in $50 \mathrm{mM}$ acetate sodium buffer ( $\mathrm{pH} 5.2$ ) containing $0.3 \mathrm{M} \mathrm{NaCl}$ at a flow rate of $0.5 \mathrm{ml} / \mathrm{min}$. The absorbance was measured at $280 \mathrm{~nm}$. Fractions containing enzyme activity were concentrated, desalted using Amicon Ultra-15 10000 MWCO (MILLIPORE), and filtered using a syringe filter (ADVANTEC ${ }^{\circ}$ DISMIC $-25 \mathrm{cs}, 0.2 \mu \mathrm{m}$, Toyo Roshi Kaisha, Ltd.). The filtrate $(100 \mu \mathrm{l})$ was mixed with $900 \mu \mathrm{l}$ of $50 \mathrm{mM}$ Na-phosphate buffer ( $\mathrm{pH} 7.1$ ) containing $1.3 \mathrm{M}$ ammonium sulfate. The mixture $(1 \mathrm{ml})$ was applied to a hydrophobic interaction column (Phenyl Superose HR 5/5; GE Healthcare UK Ltd.) with a linear gradient $(1.2-0 \mathrm{M})$ of ammonium sulfate in $50 \mathrm{mM}$ Na-phosphate buffer $(\mathrm{pH} \mathrm{7.1)}$ at a flow rate of $0.5 \mathrm{ml} / \mathrm{min}$. Fractions containing enzyme activity were concentrated, desalted, and filtered as described above. Protein concentration was measured with a protein assay kit (Bio-Rad Laboratories, Hercules, CA, USA).

\section{Enzyme $\mathrm{pH}$ and temperature properties}

Optimal pH for enzyme activity was determined at $30^{\circ} \mathrm{C}$ for $30 \mathrm{~min}$ with beechwood xylan as substrate using several $15 \mathrm{mM}$ buffers: sodium acetate- $\mathrm{HCl}$ ( $\mathrm{pH} 2.0-4.0)$, sodium acetate ( $\mathrm{pH} 4.0-5.2)$, sodium phosphate ( $\mathrm{pH} 5.2-$ 6.8), and Tris- $\mathrm{HCl}$ ( $\mathrm{pH} 6.8-8.0$ ). To estimate $\mathrm{pH}$ stability, the enzyme solutions were incubated at $\mathrm{pH}$ ranging from 2.0 to 8.0 using the above buffers for $1 \mathrm{~h}$ at $30^{\circ} \mathrm{C}$. The residual xylanase activity was then determined at $\mathrm{pH}$ 5.2. Optimal temperature for activity was determined at $\mathrm{pH}$ 5.2 for $30 \mathrm{~min}$ at different temperatures $\left(30-70^{\circ} \mathrm{C}\right)$ with beechwood xylan as substrate. Thermostability was estimated by incubating the enzyme sample in water bath at $30-70^{\circ} \mathrm{C}$ for $30 \mathrm{~min}$. After heat treatment, samples were chilled on ice and residual xylanase activity was determined as described above.

\section{Xylan hydrolysis and end product analysis}

Purified PaXynG was used to determine the end products of beechwood xylan hydrolysis. The reactions were conducted at $\mathrm{pH} 5.2$ and $30^{\circ} \mathrm{C}$. After incubations at 10, 20, and $30 \mathrm{~min}$, the reactions were stopped by boiling. The hydrolysis products were separated by thin-layer chromatography (TLC) according to the method of La Grange et al. (2001). Samples of the reaction mixtures, containing $20 \mu \mathrm{g}$ of xylose-equivalent sugars, were applied to a TLC sheet (Silica gel $60 \mathrm{~F}_{254}$, Merck, Darmstadt, Germany) that was placed in a chamber containing 7:1:2 solvent 
mixture of $n$-propanol:ethanol: $\mathrm{H}_{2} \mathrm{O}$. A xylooligosaccharides standard mixture $(20 \mu \mathrm{g} / \mu \mathrm{l})$ containing xylose (X1), xylobiose (X2), xylotriose (X3), and xylotetraose (X4) was also used. The sugar spots were identified by spraying $1 \%$ anthrone dissolved in $75 \%$ sulfuric acid, followed by heating (Morita et al. 2010). Hydrolyzed xylose was measured using a D-xylose assay kit (Megazyme, Ireland) according to the manufacturer's manual.

\section{Jar-fermentor cultivation}

Pseudozyma antarctica GB-4(0) was used for the jarfermentor cultivation experiments. A 30-ml pre-culture was grown in a $300-\mathrm{ml}$ flask at $30^{\circ} \mathrm{C}$ with rotary shaking at $200 \mathrm{rpm}$ for $24 \mathrm{~h}$ in YM medium. This culture was then used to inoculate the 5-1 jar fermentor containing 31 of PaXyn production medium [0.2\% yeast extract, $\begin{array}{llllll}0.2 \% & \mathrm{NaNO}_{3}, & 0.2 \% & \left(\mathrm{NH}_{4}\right)_{2} \mathrm{SO}_{4}, 0.04 \% & \mathrm{KH}_{2} \mathrm{PO}_{4}, 0.04 \%\end{array}$ $\mathrm{MgSO}_{4} \cdot 7 \mathrm{H}_{2} \mathrm{O}$, and $2 \%$ xylose]. Batch cultivation was performed until all the xylose was depleted (around $24 \mathrm{~h}$ ). Then, xylose-fed-batch cultivation was performed to induce PaXynG production by adding fed medium $[0.2 \%$ yeast extract, $0.085 \%$ YNB w/o amino acid and ammonium sulfate (Difco) and 20\% xylose] at a feeding rate of $500 \mathrm{ml} /$ day using a peristaltic pump. Xylose and other nutrients were separately autoclaved $\left(121^{\circ} \mathrm{C}, 20 \mathrm{~min}\right)$.

LBDW derived from rice straw hydrolysate was kindly provided by Biomaterial in Tokyo Co., Ltd. The concentration of xylose $(6.7 \%)$ was measured with a D-xylose assay kit (Megazyme, Ireland). Analysis of LBDW using standard methods (APHA et al. 1998) showed the following components: dissolved organic carbon (DOC), $52,000 \mathrm{mg} / \mathrm{l}$, dissolved total nitrogen (DTN), $550 \mathrm{mg} / \mathrm{l}$, and dissolved total phosphorus (DTP), $600 \mathrm{mg} / \mathrm{l}$. It has a $\mathrm{pH}$ of 4.2 . The pre-culture $(30 \mathrm{ml})$ prepared as described above was inoculated into 2-l of 4-times-diluted LBDW. After $24 \mathrm{~h}$ cultivation, LBDW was continuously added at a rate of 1 l/day using a peristaltic pump.

The cultivation conditions were as follows: aeration rate was 2 LPM; agitation value was $500 \mathrm{rpm}$; dissolved oxygen (DO) was maintained at around $25 \%$ of saturated value; $\mathrm{pH}$ was controlled at 6.0 with $14 \%$ ammonia solution, which also provided a nitrogen source for the culture; temperature was $30^{\circ} \mathrm{C}$.

\section{Accession number}

The accession number of the genomic sequence encoding PaXynG of P. antarctica GB-4(0) registered in DBBJ is $\mathrm{AB} 901085$.

\section{Results}

Production of xylanase from $P$. antarctica GB-4(0)

When cultivated in flask with modified FMM with $8 \%$ xylose, $P$ antarctica GB-4(0) produces a 33-kDa unknown protein (Figure 1b, indicated by the arrow). Productions of some xylanases are reported to have been induced by xylose (Furukawa et al. 2009; Gielkens et al. 1999; Masaki et al. 2012; Dobberstein and Emeis 1989). Among the Pseudozyma species found to have the ability to secrete xylanases are P. hubiensis NCIM3574 (Adsul et al. 2009) and P. brasiliensis sp. nov. strain GHG001 (Borges et al. 2014). These findings led us to speculate that the 33-kDa unknown protein produced by $P$. antarctica strains could also be xylanases. With this assumption, we investigated the time course of xylanase activity of $P$. antarctica GB-4(0) culture.

Xylanase activity was detected in the culture and was found to have gradually increased with time (Figure 1a). At $96 \mathrm{~h}, 49.9 \mathrm{U} / \mathrm{ml}$ of xylanase activity and cell growth

\section{a}

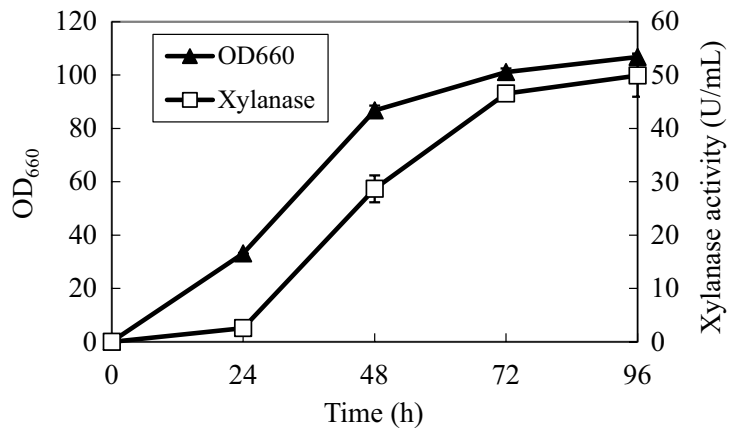

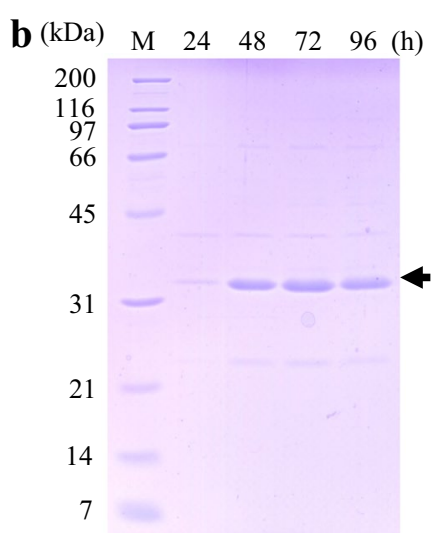

Figure 1 Time course of xylanase (PaXynG) production by P. antarctica GB-4(0) with modified FMM containing 8\% xylose in flask cultivation. a Cell growth (closed triangles) and xylanase (open squares) production. Each result is the average of three different experiments. Error bars show standard deviations. b SDS-PAGE of supernatants $(5 \mu \mathrm{l})$ periodically sampled from the flask cultivation. The arrow at $33 \mathrm{kDa}$ indicates PaXyn bands. 
with an $\mathrm{OD}_{660}$ of 106.7 were obtained (Figure 1a). Simultaneously, the intensity of the $33-\mathrm{kDa}$ band of GB-4(0) on the SDS-PAGE gel increased with time (Figure 1b). Xylan, being the substrate of xylanases, generally induces xylanase production by microorganisms. However, with modified FMM using 8\% xylan instead of xylose under the same cultivation conditions described previously, $P$. antarctica GB-4(0) showed less xylanase activity (14.0 U/ $\mathrm{ml})$. The 33-kDa band was also detected in this culture by SDS-PAGE analysis (data not shown).

\section{Nucleic acid and amino acid analysis of the two xylanases} Another $P$. antarctica strain, T-34, also produced xylanase $(18.5 \mathrm{U} / \mathrm{ml})$ under the same conditions with xylose, and the 33-kDa unknown protein band was also detected on SDS-PAGE gel (data not shown). In order to identify the xylanase of strain GB-4(0), we referred to the DNA sequence of the gene that encodes the 33-kDa unknown protein of strain T-34 based on the database for its whole genome sequence (Morita et al. 2013).

The N-terminal sequence (amino acids 35-44 after cleavage of a signal peptide) of the $33-\mathrm{kDa}$ unknown protein from $P$. antarctica $\mathrm{T}-34$ indicated that it corresponded to a gene with accession No. DF196774 (GAC73192.1) in the genome of P. antarctica T-34. This gene contains the putative promoter region (600 bp), 1,026-bp ORF, the putative terminator region (300 bp), and one putative intron (624-688) comprised of 64 nucleotides.

The deduced amino acid sequence contains 341 amino acid residues (accession No. M9ME65) and the predicted size of the mature protein (307 amino acid residues, $32.9 \mathrm{kDa}$ ) is almost the same as that estimated by SDSPAGE electrophoresis. A search of DDBJ (http://www. ddbj.nig.ac.jp/) yielded a number of xylanases belonging to glycosyl hydrolases (GH) 10 family, whose amino acid sequences are $57-77 \%$ identical to the $33-\mathrm{kDa}$ unknown protein of $P$. antarctica T-34 (Table 2). These results lend support to our speculation that the $33-\mathrm{kDa}$ unknown protein is also a xylanase (PaXyn).

The genomic sequence encoding PaXynT of $P$. antarctica T-34, was used to design primers for the amplification of the genomic sequence encoding PaXynG of $P$. antarctica GB-4(0). A single PCR product was obtained and sequenced (1,954 bp, accession No. AB901085). The nucleotide sequence encoding PaXynG was found to be 91\% identical to that encoding PaXynT over the range -600 to 1,390 of T-34. The deduced amino acid sequence (341 amino acid residues) of PaXynG is 98\% identical to that of PaXynT.

\section{Purification and characterization of PaXynG}

The PaXynG was purified by gel-chromatography and hydrophobic interaction-chromatography as described in Table 3. Purification to homogeneity was confirmed by observation of a single 33-kDa band on a silver stained SDS-PAGE gel (Figure 2). Its specific activity was about $52 \mathrm{U} / \mathrm{mg}$.

Its optimal $\mathrm{pH}$ was 5.2, and the optimal temperature was $50^{\circ} \mathrm{C}$ (Table 4 ). It was stable over a wide $\mathrm{pH}$ range (3.0-8.0), retaining $97 \%$ of their original activities after $1 \mathrm{~h}$ incubation (data not shown). The temperature at which the enzyme lost half of their activity (a measure of thermostability) was $57^{\circ} \mathrm{C}$. It had no $\beta$-xylosidase activity. The same results were obtained with purified PaXynT (Table 4).

A TLC analysis of products revealed that purified PaXynG hydrolyzed beechwood xylan to xylose (X1), xylobiose (X2), xylotriose (X3), xylotetraose (X4), and other xylooligosaccharides (Figure 3 ). The released xylose was confirmed by detection with D-xylose assay kit (data not shown).

Table 2 Percentages of sequence identity between PaXynT of P. antarctica T-34 and other xylanases

\begin{tabular}{|c|c|c|c|c|}
\hline Microorganism & Putative function & Accession no. & Identity (\%) & Protein size (aa) \\
\hline P. antarctica $\mathrm{T}-34$ & Endo-1,4- $\beta$-D-xylanase & M9ME65 & - & 341 \\
\hline Ustilago hordei & Probable endo-1,4- $\beta$-xylanase & I2FWP8 & 77 & 342 \\
\hline Sporisorium reilianum & Probable endo-1,4- $\beta$-xylanase & E7A3D3 & 75 & 343 \\
\hline Aspergillus oryzae & Endo-1,4- $\beta$-xylanase F3 & Q96VB6 & 65 & 323 \\
\hline Aspergillus sojae & Endo-1,4- $\beta$-D-xylanase & Q9P955 & 64 & 323 \\
\hline Penicillium oxalicum & Endo-1,4- $\beta$-xylanase & E1B2N4 & 61 & 330 \\
\hline Paecilomyces aerugineus & Endo- $\beta$-1,4-xylanase & G8ZAH1 & 61 & 326 \\
\hline Penicillium citrinum & Endo-1,4- $\beta$-xylanase & B1B533 & 60 & 327 \\
\hline Aspergillus terreus & Probable endo-1,4- $\beta$-xylanase $C$ & Q0CBM8 & 59 & 326 \\
\hline Thermoascus aurantiacus & Endo-1,4- $\beta$-xylanase & P23360 & 57 & 329 \\
\hline
\end{tabular}


Table 3 Purification of $P$. antarctica GB-4(0) xylanase (PaXynG)

\begin{tabular}{lccccc}
\hline & Total protein $(\mathbf{m g})$ & Total activity $(\mathbf{U})$ & Specific activity $(\mathbf{U} / \mathbf{m g})$ & Yield (\%) & Purification (fold) \\
\hline Crude culture & 90.0 & $1,287.2$ & 14.3 & 100 & 1 \\
G3000SW $_{\text {XL }}$ & 18.0 & 924.8 & 51.4 & 71.8 & 3.6 \\
Phenyl Superose HR 5/5 & 5.9 & 306.0 & 52.3 & 23.8 & 3.7 \\
\hline
\end{tabular}

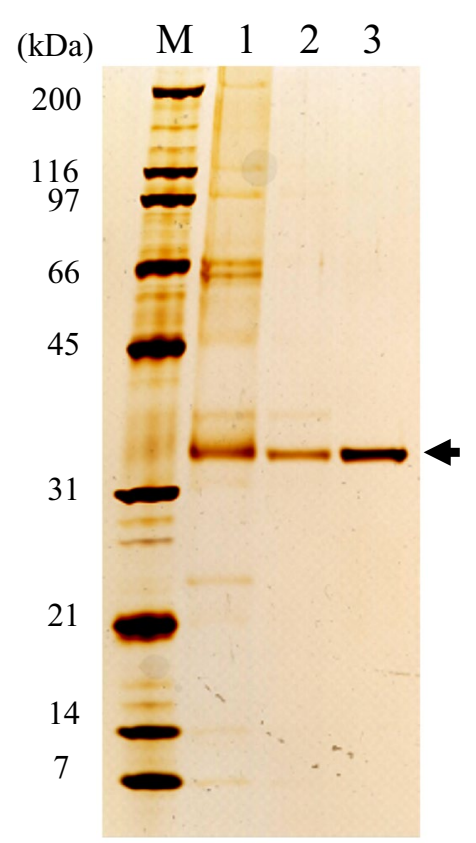

Figure 2 SDS-PAGE of PaXynG of P. antarctica GB-4(0). Lane $M$ molecular standard, lane 1 culture medium, lane 2 TSK-Gel G3000SW $\mathrm{XL}_{\mathrm{L}}$-purified PaXynG, lane 3 Phenyl Superose HR 5/5-purified PaXynG.

Table 4 Biochemical characterization of PaXyns of $P$. antarctica T-34 and GB-4(0)

\begin{tabular}{lll}
\hline Biochemical characters & PaXynT of T-34 & PaXynG of GB-4(0) \\
\hline Optimal pH & 5.2 & 5.2 \\
Optimal temperature & $50^{\circ} \mathrm{C}$ & $50^{\circ} \mathrm{C}$ \\
pH stability & $3.0-8.0$ & $3.0-8.0$ \\
Thermostability & $57^{\circ} \mathrm{C}$ & $57^{\circ} \mathrm{C}$ \\
$\beta$-Xylosidaze activity & $\mathrm{ND}$ & $\mathrm{ND}$ \\
\hline
\end{tabular}

ND not detected.

\section{Production of highly concentrated PaXynG} by xylose-fed-batch cultivation using a jar-fermentor Because $P$. antarctica GB-4(0) produced larger amounts of PaXyn than T-34 in flask cultivation, GB-4(0) was used in jar fermentor cultivation. After the initial low concentration of xylose ( $2 \%$ ) was consumed $(24 \mathrm{~h})$, xylose was fed into the PaXyn production medium. This resulted in

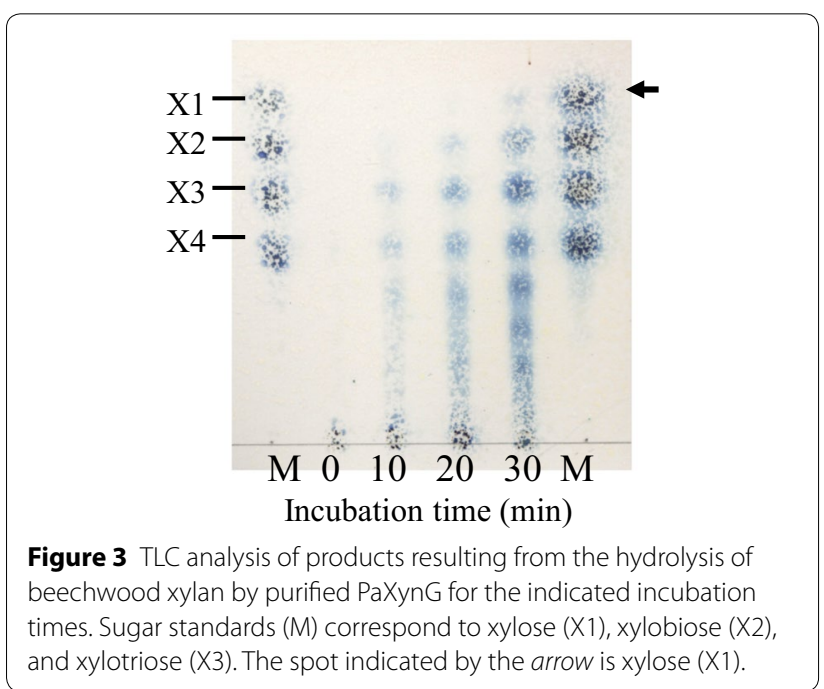

a DO value around $50 \%$ of saturation (data not shown); and 232.4 U/ml (corresponding to $4.5 \mathrm{~g} / \mathrm{l}$ ) of PaXynG was obtained after $72 \mathrm{~h}$ (Figure 4).

\section{Simultaneous production of PaXynG from LBDW} during its treatment using a jar-fermentor

In a preliminary examination, we found that $P$. antarctica GB-4(0) and T-34 could not grow on LBDW unless it was diluted 4-5 times with water (data not shown).

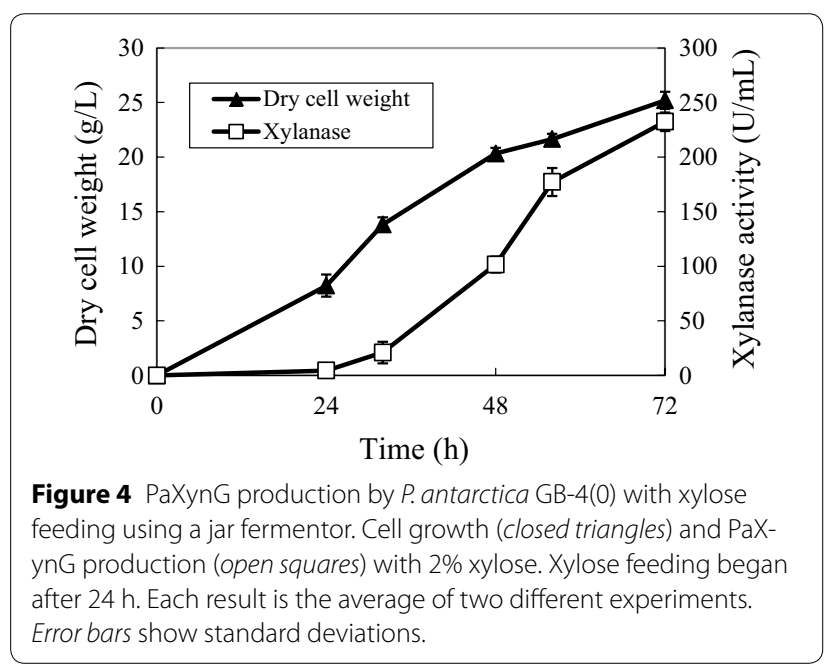


Thus, LBDW-fed-batch cultivation was initially carried out with 4-times-diluted LBDW. After the initial carbon sources, that could be utilized by $P$. antarctica GB-4(0), was consumed ( $24 \mathrm{~h}$ ), non-diluted LBDW was fed into the culture using a peristaltic pump. This resulted in a DO value around $60 \%$ of saturation (data not shown); $17.3 \mathrm{U} / \mathrm{ml}(0.3 \mathrm{~g} / \mathrm{l})$ of PaXynG was obtained after $72 \mathrm{~h}$ (Figure 5a). PaXynG was detected as a $33-\mathrm{kDa}$ band on a CBB stained SDS-PAGE gel (Figure $5 b$ ).

In terms of wastewater treatment, over $99 \%$ of the xylose content of the LBDW was consumed after 72-h cultivation of $P$. antarctica GB-4(0). The resulting effluent DOC and DTP were 12,000 and $48 \mathrm{mg} / \mathrm{l}$, respectively. The total amount of influent DOC $((26,000 \mathrm{mg}+104,000 \mathrm{mg}) / 4 \mathrm{l})$ and DTP $((300 \mathrm{mg}+1,200 \mathrm{mg}) / 4 \mathrm{l})$ were calculated as 32,500 and $375 \mathrm{mg} / \mathrm{l}$, respectively. Thus, considering these values,
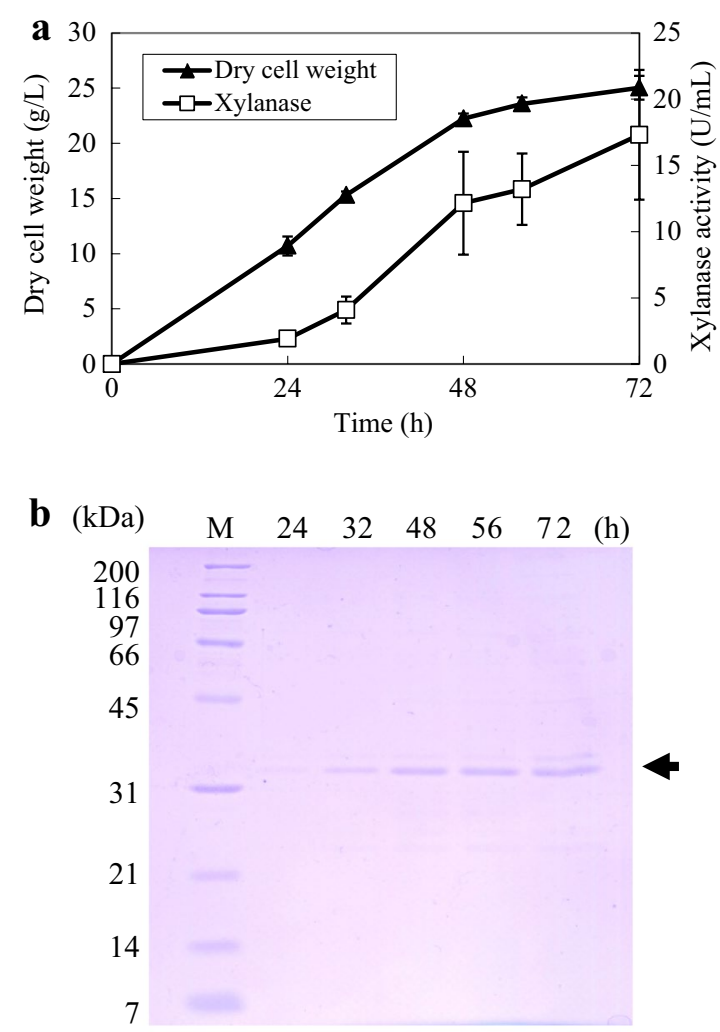

Figure 5 LBDW-fed cultivation of $P$. antarctica GB-4(0) using a jar fermentor. a Cell growth (closed triangles) and PaXynG production (open squares) with 4-times-diluted LBDW. LBDW feeding began after $24 \mathrm{~h}$. Each result is the average of two different experiments. Error bars show standard deviations. b SDS-PAGE of supernatants $(5 \mu \mathrm{l})$ periodically sampled from the jar fermentor cultivation. The arrow at $33 \mathrm{kDa}$ indicates PaXynG bands. the removal ratios were calculated to be 63 and $87 \%$, respectively. Day removal ratio of DOC $\left(6.8 \mathrm{~kg} / \mathrm{m}^{3} /\right.$ day $)$ was much higher compared to that removed by activated sludge (0.5-1.0 kg/m $/ \mathrm{m}^{3} /$ day) (Watanabe et al. 2013a).

\section{Discussion}

In this study, we attempted to produce useful materials from LBDW by cultivating $P$. antarctica during treatment of this wastewater. We first identified the highly-secreted $33-\mathrm{kDa}$ unknown proteins in the culture of $P$. antarctica GB-4(0) and T-34 cultivated with xylose to be novel xylanases (PaXyns). Next, we determined the genomic sequence for PaXyn of P. antarctica T-34 (PaXynT) using the annotated whole genome sequence (Morita et al. 2013). P. antarctica GB-4(0) produced higher PaXyn than $P$. antarctica T-34 (Figure 1). Using the sequence encoding PaXynT, we successfully identified the genomic sequence for PaXyn of $P$. antarctica GB-4(0) (PaXynG). Xylanases are classified into two substantial groups, GH 10 and 11 family, on the basis of their structures (Kimura et al. 2010). The sequence similarity of these PaXyns indicated that they belong to GH10 family xylanases which exhibit higher affinity for shorter liner $\beta-1,4-$ xylooligosaccharides than GH11 family xylanases (Biely et al. 1997).

The optimal $\mathrm{pH}$, optimal temperature, $\mathrm{pH}$ stability, and thermostability of the both PaXyns are almost the same (Table 4). They produced xylose (X1), xylobiose (X2), xylotriose (X3), and other xylooligosaccharides from xylan (Figure 3 ) and did not have any $\beta$-xylosidase activity (Table 4). These data indicate that they are endoxylanases (1,4- $\beta$-D-xylan xylanohydrolases (EC 3.2.1.8)). Since these PaXyns did not have any $\beta$-xylosidase activity, they could not hydrolyze xylobiose to xylose. Thus, the detected xylose might have resulted from the hydrolysis of xylotriose and other xylooligosaccharides by PaXyns. With this result, it is possible that just like the other endoxylanases (Biely 1985), PaXyns could be employed in saccharizing lignocellulosic biomass materials for the production of useful products.

The production of PaXyns was strongly induced by xylose; a yield of $4.5 \mathrm{~g} / \mathrm{l}$ of PaXynG was produced by $P$. antarctica GB-4(0) in $72 \mathrm{~h}(0.0625 \mathrm{~g} / \mathrm{l} / \mathrm{h})$ with xylose fed-batch cultivation using a jar fermentor (Figure 4). This yield is comparable with $8.1 \mathrm{~g} / \mathrm{l}$ of recombinant xylanase (PtxynA) of Paecilomyces thermorphila produced by a methylotrophic yeast Pichia pastoris in $228 \mathrm{~h}(0.0355 \mathrm{~g} / \mathrm{l} / \mathrm{h})$ with methanol fed-batch cultivation using a jar fermentor (Fan et al. 2012). The results show that $P$. antarctica is an attractive producer of native xylanase. After centrifugation, 
about 31 of supernatant was obtained from $3.5 \mathrm{l}$ of jar culture. The rate of xylanase production (produced PaXynG, $4.5 \mathrm{~g} / \mathrm{l} \times 3 \mathrm{l}=13.5 \mathrm{~g}) /($ consumed xylose, $60 \mathrm{~g}+200 \mathrm{~g}=260$ g) by this strain was calculated to be $0.052 \mathrm{~g} / \mathrm{g}$.

The hydrolysate of lignocelluloses contains not only fermentable sugars but also toxic compounds that inhibit cell growth, such as furans and organic acids (Okuda et al. 2008). Since these compounds are also generated during fermentation and distillation processes, they are expected to be present in LBDW. It is probably the reason why $P$. antarctica GB-4(0) could not grow in non-diluted LBDW. However, with LBDW fed-batch cultivation, about $0.3 \mathrm{~g} / \mathrm{l}$ of PaXynG was produced by $P$. antarctica GB-4(0) in $72 \mathrm{~h}$. After centrifugation, about 3.51 of supernatant was obtained from $4.0 \mathrm{l}$ of jar culture. This is equivalent to the xylanase production rate of about $0.006 \mathrm{~g} / \mathrm{g}$ (produced PaXynG, $0.3 \mathrm{~g} / \mathrm{l} \times 3.5 \mathrm{l}=1.05 \mathrm{~g} / \mathrm{con}$ sumed xylose of LBDW, $34.5 \mathrm{~g}+134 \mathrm{~g}=168.5 \mathrm{~g}$ ).

PaXynG production by $P$. antarctica GB-4(0) from LBDW, resulted in the removal of $63 \%$ of DOC and $87 \%$ of DTP. Because ammonia solution was added for $\mathrm{pH}$ control and nitrogen source, DTN removal ratio could not be accurately estimated. However, since the initial DTN was low, the residual DTN is expected to consist mainly of PaXynG which could be easily recovered by ultrafiltration. In the case of shochu distillery wastewater, we previously confirmed that the yeast-treated wastewater was efficiently treated by a combination of nitrification/denitrification cycle treatment and activated sludge process (Watanabe et al. 2009, 2013c). In a laboratoryscale demonstration, 50 cycles (25 days) removed $98.9 \%$ of DOC, 95.7 of DTN, and 94.1\% of DTP from barley shochu distillery wastewater (Watanabe et al. 2013c). Thus, it can be expected that the remaining DOC, DTN, and DTP of $P$. antarctica GB-4(0)-treated LBDW could also be easily removed by additional conventional wastewater treatment methods. These results indicated that xylanase production by $P$. antarctica GB-4(0) from LBDW could also contribute to the cutting of LBDW treatment cost.

\begin{abstract}
Abbreviations
COD: chemical oxygen demand; DOC: dissolved organic carbon; DO: dissolved oxygen; DTN: dissolved total nitrogen; DTP: dissolved total phosphorus; FMM: fungal minimum medium; LBDW: lignocellulosic bioethanol distillery wastewater; PaXynG: xylanase of $P$. antarctica GB-4(0); PaXynT: xylanase of $P$. antarctica T-34; SDS-PAGE: Sodium dodecyl sulfate-polyacrylamide gel electrophoresis; TLC: thin-layer chromatography
\end{abstract}

\footnotetext{
Authors' contributions

TW designed the study, carried out most of the biological studies and statistical analyses, and drafted the manuscript. KS carried out the purification of PaXyns. IS analyzed xylanase activity. TM identified genomic sequence of PaXyn of P. antarctica T-34. HK helped in the genomic sequence analysis of PaXyns. YS helped in the purification of PaXyns. HU helped in the characterization of PaXyns. MK interpreted the data and revised the manuscript. HKK conceived and planned the study, and helped in drafting the manuscript. All authors read and approved the final manuscript.
}

\section{Author details}

National Institute for Agro-Environmental Sciences (NIAES), 3-1-3 Kannondai, Tsukuba, Ibaraki 305-8604, Japan. ${ }^{2}$ Japan Society for the Promotion of Science, 1-8 Chiyoda-ku, Tokyo 102-8472, Japan. ${ }^{3}$ Research Institute for Innovation in Sustainable Chemistry, National Institute of Advanced Industrial Science and Technology (AIST), Tsukuba Central 5-2, 1-1-1 Higashi, Tsukuba, Ibaraki 305-8565, Japan. ${ }^{4}$ Bioprocess Research Institute, National Institute of Advanced Industrial Science and Technology (AIST), Tsukuba Central 6, 1-1-1 Higashi, Tsukuba, Ibaraki 305-8566, Japan.

\section{Acknowledgments}

We thank Prof. Haruyuki lefuji, Ehime University, Japan, for his helpful discussions and Mrs. Xiao-hong Cao, for technical assistance. LBDW derived from hydrolysate of rice straw was kindly provided by Biomaterial in Tokyo Co., Ltd. This work was supported by a grant of science and technology research promotion program for agriculture, forestry, fisheries and food industry; by a Research Fellowship of the Japan Society for the Promotion of Science (JSPS) for Young Scientists, and by a JSPS KAKENHI Grant (No. 23 10107).

\section{Compliance with ethical guidelines}

\section{Competing interests}

The authors declare that they have no competing interests.

Received: 9 February 2015 Accepted: 19 May 2015

Published online: 12 June 2015

\section{References}

Adsul MG, Bastwade KB, Gokhale DV (2009) Biochemical characterization of two xylanases from yeast Pseudozyma hubiensis producing only xyloologosaccharides. Bioresour Technol 100:6488-6495. doi:10.1016/j. biortech.2009.07.064

Angenent LT, Karim K, Al-Dahhan MH, Wrenn BA, Domíguez-Espinosa R (2004) Production of bioenergy and biochemical from industrial and agricultural wastewater. Trends Biotechnol 22:477-485. doi:10.1016/j. tibtech.2004.07.001

APHA, AWWA, WEF (1998) Standard methods for the examination of water and wastewater, 20th edn. American Public Health Association, Washington DC

Beg QK, Kapoor M, Mahajan L, Hoondal GS (2001) Microbial xylanase and their industrial applications: a review. Appl Microbiol Biotechnol 56:326-338. doi:10.1007/s002530100704

Biely P (1985) Microbial xylanolytic systems. Trends Biotechnol 3:286-290. doi:10.1016/0167-7799(85)90004-6

Biely P, Vršanská M, Tenkanen M, Kluepfel D (1997) Endo- $\beta$-1,4-xylanase families: differences in catalytic properties. J Biotechnol 57:151-166. doi:10.1016/S0168-1656(97)00096-5

Boekhout T (2011) Pseudozyma Bandoni emend. Boekhout (1985) and a comparison with the yeast state of Ustilago maydis (De Candolle) Corda (1842). The Yeasts, a Taxonomic study, vol 3, Fifth edn, pp 1857-1868

Borges TA, de Souza AT, Squina FM, Riaño-Pachón DM, dos Santos RAC, Machado E et al (2014) Biochemical characterization of an endoxylanase from Pseudozyma brasiliensis sp. nov. strain GHG001 isolated from the intestinal tract of Chrysomelidae larvae associated to sugarcane roots. Process Biochem 49:77-83. doi:10.1016/j.procbio.2013.10.004

Dobberstein J, Emeis CC (1989) $\beta$-Xylanase produced by Aureobasidium pullulans CBS 58475. Appl Microbiol Biotechnol 32:262-268. doi:10.1007/ BF00184971

Fan G, Katrolia P, Jia H, Yang S, Yan Q, Jiang Z (2012) High-level expression of a xylanase gene from thermophilic fungus Paecilomyces thermophila in Pichia pastoris. Biotechnol Lett 34:2043-2048. doi:10.1007/ s10529-012-0995-3

Furukawa T, Shida Y, Kitagami N, Mori K, Kato M, Kobayashi T et al (2009) Identification of specific binding sites for XYR1, a transcriptional activator of cellulolytic and xylanolytic genes in Trichoderma reesei. Fungal Genet Biol 46:564-574. doi:10.1016/j.fgb.2009.04.001

Gielkens MMC, Dekkers E, Visser J, de Graaff LH (1999) Two cellobiohydrolase-encoding genes from Aspergillus niger require D-xylose and the 
xylanolytic transcriptional activator XInR for their expression. Appl Environ Microbiol 65:4340-4345

Hatanaka C, Kobara Y (1980) Determination of glucose by a modification of Somogyi-Nelson method. Agric Biol Chem 44:2943-2949

lefuji H, Chino M, Kato M, limura Y (1996) Acid xylanase from yeast Cryptococcus sp. S-2: purification, characterization, cloning, and sequencing. Biosci Biotechnol Biochem 60:1331-1338. doi:10.1271/bbb.60.1331

Kimura T, Mizutani T, Sun J, Kawazu T, Karita S, Sakka M et al (2010) Stable production of thermotolerant xylanase B of Clostridium stercorarium in transgenic tobacco and rice. Biosci Biotechnol Biochem 74:954-960. doi:10.1271/bbb.90774

Kitamoto D, Akiba S, Hioki C, Tabuchi T (1990) Extracellular accumulation of mannosylerythritol lipids by a strain of Candida antarctica. Agric Biol Chem 54:31-36

Kitamoto HK, Shinozaki Y, Cao X, Konishi M, Morita T, Tago Ket al (2011) Phyllosphere yeasts rapidly break down biodegradable plastics. AMB Express 1:44. doi:10.1186/2191-0855-1-44

La Grange DC, Pretorius IS, Claeyseens M, van ZyI WH (2001) Degradation of xylan to D-xylose by recombinant Saccharomyces cerevisiae coexpressing the Aspergillus niger $\beta$-xylosidase $(x / n D)$ and the Trichoderma reesei xylanase II (xy/2) genes. Appl Environ Microbiol 67:5512-5519. doi:10.1128/ AEM.67.12.5512-5519.2001

Laemmli UK (1970) Cleavage of structural proteins during the assembly of the head of bacteriophage T4. Nature 277:680-685

Masaki K, Tsuchioka H, Hirano T, Kato M, Ikeda H, lefuji H (2012) Construction of a new recombinant protein expression system in the basidiomycetous yeast Cryptococcus sp. strain S-2 and enhancement of the production of a cutinase-like enzyme. Appl Microbiol Biotechnol 93:1627-1636. doi:10.1007/s00253-011-3680-x

Matsushika A, Watanabe S, Kodakari T, Makino K, Sawayama S (2008) Bioethanol production from xylose by recombinant Saccharomyces cerevisiae expression xylose reductase, NADP+-dependent xylitol dehydrogenase, and xylulokinase. J Biosci Bioeng 105:296-299. doi:10.1263/jbb.105.296

Morita T, Ito E, Fukuoka T, Imura T, Kitamoto D (2010) The role of PaAACI encoding a mitochondrial ADP/ATP carrier in the biosynthesis of extracellular glycolipids, mannosylerythritol lipids, in the basidiomycetous yeast Pseudozyma antarctica. Yeast 27:379-388. doi:10.1002/yea.1761

Morita T, Koike H, Koyama Y, Hagiwara H, Ito E, Fukuoka T et al (2013) Genome sequence of the basidiomycetous yeast Pseudozyma antarctica T-34, a producer of the glycolipid biosurfactants mannosylerythritol lipids. Genome Announc 1:e00064-13. doi:10.1128/genomeA.00064-13
Okuda N, Ninomiya K, Katakura Y, Shioya S (2008) Strategies for reducing supplemental medium cost in bioethanol production from waste house wood hydrolysate by ethanologenic Escherichia coli: inoculation size increase and coculture with Saccharomyces cerevisiae. J Biosci Bioeng 105:90-96. doi:10.1263/jbb.105.90

Pokhrel D, Viraraghavan T (2004) Treatment of pulp and paper mill wastewater-a review. Sci Total Environ 333:37-58. doi:10.1016/j. scitotenv.2004.05.017

Shinozaki Y, Morita T, Cao X, Yoshida S, Koitabashi M, Watanabe T et al (2013) Biodegradable plastic-degrading enzyme from Pseudozyma antarctica: cloning, sequencing, and characterization. Appl Microbiol Biotechnol 97:2951-2959. doi:10.1007/s00253-012-4188-8

Siso MIG (1996) The biotechnological of utilization of cheese whey: a review. Bioresour Technol 57:1-11. doi:10.1016/0960-8524(96)00036-3

Watanabe T, Masaki K, Iwashita K, Fujii T, lefuji H (2009) Treatment and phosphorus removal from high-concentration organic wastewater by the yeast Hansenula anomala J224 PAWA. Bioresour Technol 100:1781-1785. doi:10.1016/j.biortech.2008.10.006

Watanabe T, lefuji H, Kitamoto HK (2013a) Treatment of, and Candida utilis biomass production from shochu wastewater; the effects of maintaining a low pH on DOC removal and feeding cultivation on biomass production. Springerplus 2:514. doi:10.1186/2193-1801-2-514

Watanabe T, lefuji H, Kitamoto HK (2013b) Breeding of wastewater treatment yeasts having high nitrogen removal ability. J Brew Soc Jpn 108:823-829 (In Japanese)

Watanabe T, Fujii T, lefuji H, Kitamoto HK (2013c) Treatment and reutilization of Shochu wastewater using yeasts. Yousui Haisui 55:605-612 (In Japanese)

Watanabe T, Shinozaki Y, Suzuki K, Koitabashi M, Yoshida S, SameshimaYamashita Y et al (2014a) Production of a biodegradable plastic-degrading enzyme from cheese whey by the phyllosphere yeast Pseudozyma antarctica GB-4(1)W. J Biosci Bioeng 118:183-187. doi:10.1016/j. jbiosc.2014.01.007

Watanabe T, Shinozaki Y, Yoshida S, Koitabashi M, Sameshima-Yamashita Y, Fujii T et al (2014b) Xylose induces the phyllosphere yeast Pseudozyma antarctica to produce a cutinase-like enzyme which efficiently degrades biodegradable plastics. J Biosci Bioeng 117:325-329. doi:10.1016/J. jbiosc.2013.09.002

Yoshizawa K (1978) Treatment of waste-water discharged from sake brewery using yeast. J Ferment Technol 56:389-395

\section{Submit your manuscript to a SpringerOpen ${ }^{\circ}$ journal and benefit from:}

- Convenient online submission

- Rigorous peer review

- Immediate publication on acceptance

- Open access: articles freely available online

- High visibility within the field

- Retaining the copyright to your article

Submit your next manuscript at $>$ springeropen.com 